\title{
Peer review in scholarly journals: Perspective of the scholarly community - Results from an international study
}

\author{
Mark Ware \\ Mark Ware Consulting, UK \\ E-mail: mark@markwareconsulting.com
}

This summary is extracted from the report of the same title published by the Publishing Research Council (PRC) and reproduced here by kind permission of the PRC. The full report and a shorter edited version can be found on the PRC website at http://www.publishingresearch.net/PeerReview.htm.

Peer review is seen as an essential component of scholarly communication, the mechanism that facilitates the publication of primary research in academic journals. Although sometimes thought of as an essential part of the journal, it is only since the second world war that peer review has been institutionalised in the form we know it today. More recently it has come under criticism on a number of fronts: it has been said that it is unreliable, unfair and fails to validate or authenticate; that it is unstandardised and idiosyncratic; that its secrecy leads to irresponsibility on the part of reviewers; that it stifles innovation; that it causes delay in publication; and so on. Perhaps the strongest criticism is that there is a lack of evidence that peer review actually works, and a lack of evidence to indicate whether the documented failings are rare exceptions or the tip of an iceberg.

The survey reported here does not attempt directly to address the question of whether or not peer review works, but instead looks in detail at the experiences and perceptions of a large group of mostly senior authors, reviewers and editors (there is of course considerable overlap between these groups). Respondents were spread by region and by field of research broadly in line with the universe of authors publishing in the journals in the Thomson Scientific database, which covers the leading peer reviewed journals. The survey presents its findings in two broad areas: attitudes to peer review and current practices in peer review.

\section{Attitudes to peer review}

1. Peer review is widely supported. The overwhelming majority (93\%) disagree that peer review is unnecessary. The large majority ( $85 \%$ ) agreed that peer review greatly helps scientific communication and most $(83 \%)$ believe that without peer review there would be no control.

2. Peer review improves the quality of the published paper. Researchers overwhelmingly $(90 \%)$ said the main area of effectiveness of peer review was in improving the quality of the published paper. In their own experience as authors, $89 \%$ said that peer review had improved their last published paper, both in terms of the language or presentation but also in terms of correcting scientific errors. 
3. There is a desire for improvement. While the majority (64\%) of academics declared themselves satisfied with the current system of peer review used by journals (and just 12\% dissatisfied), they were divided on whether the current system is the best that can be achieved, with $36 \%$ disagreeing and $32 \%$ agreeing. There was a very similar division on whether peer review needs a complete overhaul. There was evidence that peer review is too slow (38\% were dissatisfied with peer review times) and that reviewers are overloaded (see \#13 below).

4. Double-blind review was preferred. Changes to peer review in recent years (such as the growth of double-blind review, and the introduction of open and post-publication review) have attempted to improve the system. Asked which of the four peer review types was their most preferred option, there was a preference for double-blind review, with $56 \%$ selecting this, followed by $25 \%$ for single-blind, $13 \%$ for open and 5\% for post-publication review. Open peer review was an active discouragement for many reviewers, with $47 \%$ saying that disclosing their name to the author would make them less likely to review.

5. Double-blind review was seen as the most effective. Of the four types of peer review discussed, double-blind review had the most respondents $(71 \%)$ who perceived it to be effective, followed (in declining order) by single-blind (52\%), post-publication (37\%) and open peer review (26\%). Respondents did not have personal experience of all types of review and tended to rate more highly the systems they had experienced. It is notable, though, that although $37 \%$ of respondents said that post-publication review was effective, only $8 \%$ had had experience of it as authors.

6. Double-blind review faces some fundamental objections. Double-blind review was primarily supported because of its perceived objectivity and fairness. Many respondents, including some of those supporting double-blind review, did however point out that there were great difficulties in operating it in practice because it was frequently too easy to identify authors from their references, type of work or other internal clues.

7. Post-publication review was seen as a useful supplement to formal peer review. In terms of recent developments facilitated by technology advances, some $37 \%$ thought that post-publication review was effective but only $5 \%$ preferred it over other approaches. It is clear that this was because researchers tended to see it as a useful supplement to formal peer review rather than a replacement for it (53\% agreed compared to $23 \%$ disagreeing). Interestingly, they saw this usefulness despite a clear view that it tends to encourage instant reactions and discourage thoughtful review.

8. No support for replacing peer review with metrics. There was strong opposition to replacing peer review with post-publication ratings or usage or citation statistics to identify good papers, with only 5-7\% of respondents supporting these approaches.

9. Mixed support for review of authors' data. A majority of reviewers (63\%) and editors (68\%) say that it is desirable in principle to review authors' data. Perhaps surprisingly, a majority of reviewers (albeit a small one, 51\%) said that they would be prepared to review authors' data themselves, compared to only $19 \%$ who disagreed. This was despite $40 \%$ of reviewers (and $45 \%$ of editors) saying that it was unrealistic to expect peer reviewers to review authors' data. Given that many reviewers also reported being overloaded, we wonder, however, whether they would still be as willing when it actually came to examine the data.

10. Limited support for payment for reviewers. Respondents were divided on whether reviewers should be paid, with $35 \%$ in favour and $40 \%$ against payment. A majority, however, supported the proposition that payment would make the cost of publishing too expensive (52\% for, $18 \%$ against) and the large majority of reviewers (91\%) said that they reviewed to play their part as a member of the academic community. 


\section{Current practices in peer review}

11. Single-blind review was the most commonly experienced. The average respondent had published 60 papers in their career to date, suggesting they were fairly experienced and productive researchers, and 8 papers in the last 24 months. As authors, respondents' experience of peer review was mainly of single-blind reviewing ( $84 \%$ said they had experienced this kind of review), followed at some distance by double-blind reviewing (44\%). Less than a quarter (22\%) reported experience of open peer review, while experience of post-publication review was limited to $8 \%$ of respondents.

12. Longer review times was a cause of dissatisfaction. Authors said the peer review of their last published paper took an average of 80 days. They were evenly balanced on whether or not this was satisfactory. There was a clear correlation between the reported time taken for peer review and the author's satisfaction: $67 \%$ were satisfied provided the time was under 30 days, but this dropped to $10 \%$ for 3-6 months, and to $9 \%$ for longer than 6 months.

13. The most productive reviewers were overloaded. Some $90 \%$ of authors were also reviewers, acting regularly for about 3.5 journals and a further 4.2 journals occasionally. They reported reviewing an average of 8 papers in the last 12 months, compared to the maximum of 9 that they said they were prepared to review. Active reviewers, defined as those doing 6 or more reviews in the last 12 months, completed an average of 14 reviews per year, nearly twice the overall figure. This means that although Active reviewers make up $44 \%$ of all reviewers, they are responsible for $79 \%$ of all reviews. So when this group reports it is over-loaded - doing 14 reviews per year compared to their preferred maximum of 13 - there is clearly a problem.

14. About $20 \%$ of invitations to review are declined. As well as completing 8 reviews per year, the average reviewer declined about 2 invitations to review, mainly because of a lack of time. Active reviewers, despite doing more reviews, if anything decline slightly fewer invitations proportionately.

15. The average review takes 5 hours and is completed in 3-4 weeks. Reviewers say that they took about 24 days (elapsed time) to complete their last review, with $85 \%$ reporting that they took 30 days or less. They spent a median 5 hours (mean 9 hours) per review.

16. Altruistic reasons for reviewing were preferred over self-interested ones. Substantially the most popular given was "playing your part as a member of the academic community". Self-interested reasons such as "to enhance your reputation or further your career" or "to increase the chance of being offered a role in the journal's editorial team" were much less frequently advanced.

17. The average acceptance rate was $50 \%$. Editors reported that the average acceptance rate for their journals was about $50 \%$, which is consistent with other studies. About $20 \%$ are rejected prior to review (either because of poor quality $(13 \%)$ or being out of scope $(8 \%)$ ) and another $30 \%$ are rejected following review. Of the 50\% accepted, $40 \%$ are accepted subject to revision. Acceptance rates were lower in humanities and social sciences, and higher in physical sciences/engineering journals.

18. Use of online submissions systems. Three quarters of editors ( $76 \%$ ) reported that their journal used an online manuscript submission and tracking system. Their use was most common in life sciences $(85 \%)$ and least common in humanities and social sciences $(51 \%)$.

19. Access to journals literature. Some $69 \%$ of respondents described their access to the journals literature as good or excellent, with $7 \%$ describing it as poor or very poor. This probably represents an improvement in overall access compared to the CIBER 2004 survey [1], which reported $61 \%$ with 
good/excellent and $10 \%$ poor/very poor (though a different geographical distribution of responses makes direct comparison difficult).

The survey thus paints a picture of academics committed to peer review, with the vast majority believing that it helps scientific communication and in particular that it improves the quality of published papers. They are willing to play their part in carrying out review, though it is worrying that the most productive reviewers appear to be overloaded. Many of them are in fact willing to go further than at present and take on responsibility for reviewing authors' data.

Within this picture of overall satisfaction there are, however, some sizeable pockets of discontent. This discontent does not always translate into support for alternative methods of peer review; in fact some of those most positive about the benefits of peer review were also the most supportive of post-publication review. Overall, there was substantial minority support for post-publication review as a supplement to formal peer review, but much less support for open review as an alternative to blinded review.

\section{Reference}

[1] I. Rowlands, D. Nicholas and P. Huntingdon, Scholarly Communication in the Digital Age: What Do Authors Want?, Centre for Information Behaviour (CIBER), 2004. 